\title{
AVALIAÇÃO SENSORIAL E FÍSICO-QUÍMICA DE DOCE DE LEITE PASTOSO CONTENDO DIFERENTES CONCENTRAÇÕES DE SORO DE LEITE
}

\section{Sensory and physicochemical evaluation of pasty dulce de leche containing different concentrations of whey}

\author{
Maria Eduarda Vilela ${ }^{*}$, Juliana Martins Braz ${ }^{1}$, Mirelle Maira Mariano ${ }^{1}$, Nathalie Goes \\ Bullhões ${ }^{l}$, Douglas Castro dos Santos ${ }^{l}$, Mariana Borges de Lima Dutra ${ }^{l}$
}

\section{RESUMO}

A fabricação de queijos gera soro como subproduto, porém a maioria dos laticínios o descarta, sem o correto tratamento para este resíduo, gerando grande impacto ambiental. O doce de leite é uma sobremesa láctea de grande importância para a economia brasileira e, portanto, a utilização do soro como substituição parcial do leite é um fator preponderante no que se refere ao aumento da lucratividade dessas empresas, bem como à diminuição dos problemas ambientais relacionados. Sendo assim, o objetivo do trabalho foi compreender a influência nas características organolépticas da substituição parcial do leite integral por soro de leite em formulações de doce de leite e determinar suas características físico-químicas. Foram realizadas seis formulações com diferentes concentrações de soro, para posterior avaliação sensorial quanto aos atributos de aparência, sabor, textura, aroma e impressão global, bem como o teste do ideal para gosto doce, consistência e intenção de compra. As análises físico-químicas realizadas foram as de umidade, proteína, textura, ${ }^{\circ}$ Brix, lipídios, colorimetria e pH. A partir da análise dos resultados, observou-se que os doces mais aceitos sensorialmente foram as formulações 3 e 4, com substituição parcial do leite por $33,33 \%$ e $41,67 \%$, respectivamente. Diante destes resultados, podemos afirmar que a substituição parcial de leite por soro de leite é uma alternativa viável dentro do intervalo compreendido pelas amostras mais aceitas.

Palavras-chave: produtos lácteos, reaproveitamento, teste do ideal, mapa de preferência.

1 Instituto Federal do Sul de Minas Gerais, Campus Inconfidentes, Praça Tiradentes, 416, Centro, 37576 000, Inconfidentes, MG, Brasil. E-mail: vilelamariaeduarda@gmail.com

* Autor para correspondência

Recebido / Received: 26/06/2019

Aprovado / Approved: 10/03/2020 


\begin{abstract}
Cheese production generates whey as a byproduct, but most of dairy companies discard it without the correct treatment for this residue, creating a large environmental impact. Dulce de leche is a dairy dessert of great importance for the Brazilian economy and, therefore, the use of whey as a partial replacement of milk is a preponderant factor in the increase of the profitability of these industries, and the reduction of the environmental problems related. Therefore, the objective of this work was to understand the influence on the organoleptic characteristics of the partial substitution of whole milk by whey in dulce de leche formulations and to determine their physicochemical characteristics. Six formulations with different concentrations of whey were developed for further sensorial evaluation of the attributes of appearance, taste, texture, aroma, and overall impression, as well as the ideal test for sweet taste and consistency and purchase intention. The physicochemical analyses were done considering moisture, protein, texture, ${ }^{\circ}$ Brix, lipids, colorimetry, and $\mathrm{pH}$. From the analysis of the results, it was observed that the most accepted formulations of dulce de leche were the 3 and 4, with partial replacement of milk by 33,33\% and 41,67\%, respectively. Considering these results, we can affirm that the partial substitution of milk by whey is a viable alternative within the range comprised by the most accepted samples.
\end{abstract}

Keywords: dairy products, reuse, ideal test, preference map.

\section{INTRODUÇÃO}

O leite é um alimento com alto teor nutritivo, obtido através das glândulas mamárias de animais, sendo majoritariamente utilizado no Brasil, o de vaca. Apresentase como um fluido viscoso, de fase líquida com partículas em suspensão (gotículas de gordura e micelas de caseína), o que gera uma emulsão natural estável em situações normais de temperatura ou de refrigeração. Constituídos predominantemente por água $(87,30 \%)$, e macronutrientes como lactose $(4,90 \%)$, gordura $(3,80 \%)$, proteínas $(3,30 \%)$ e minerais $(0,72 \%)$ (SGARBIER, 2004).

O soro é um subproduto da fabricação de queijos que pode representar de $80 \%$ a $90 \%$ do volume total do leite utilizado durante sua produção. Este é composto por água e aproximadamente $55 \%$ dos nutrientes do leite, sendo eles, minerais, proteínas solúveis, lactose, vitaminas e uma baixa quantidade de gordura, que, após a coagulação do leite, ficam retidas no soro (ALVES et al., 2014).

Apesar de sua importância como fonte nutritiva, o soro de leite apresenta sério problema de poluição ambiental, visto que aproximadamente $50 \%$ são lançados diretamente em curso d'água (SILVA et al., 2003). Isso propicia uma grande agressão ao meio ambiente causada pelos efluentes líquidos gerados e um desperdício de um subproduto que pode ser utilizado para enriquecer nutricionalmente preparações alimentares.

Ainda segundo ALVES et al. (2014), muitos laticínios ainda consideram o soro de leite um efluente, que, se não for tratado corretamente, gera poluição ambiental (devido a concentração de matéria orgânica), e para diminuir este impacto, existe a possibilidade de reaproveitamento.

Diante destes fatores, a proposta deste estudo é de grande importância ambiental, econômica e tecnológica, uma vez que as indústrias de laticínios podem se beneficiar 
pela oportunidade de aproveitamento do soro, evitando que seja lançado nos mananciais, diminuindo a agressão ao meio ambiente (FERRARI et al., 2013).

Apesar da viabilidade desta substituição, faz se necessária uma prévia análise sensorial, procedimento este, segundo o Instituto Adolfo Lutz (ZENEBON et al., 2008), que permite a interpretação das propriedades intrínsecas dos produtos, como o aroma, a textura, o sabor e a cor, entre outros. A análise sensorial se utiliza de estudos estatísticos em função das respostas transmitidas pelos indivíduos, o que por sua vez, possibilita a avaliação da aceitabilidade de um produto frente a variação de algum ingrediente, mudança no processamento ou mesmo desenvolvimento de novos produtos (TEIXEIRA, 2009).

Este trabalho teve como objetivo compreender a influência da substituição parcial do leite integral por soro de leite em formulações de doce de leite em suas características organolépticas, apresentando também os teores de alguns macrocomponentes e características físico-químicas dos produtos obtidos.

\section{MATERIAL E MÉTODOS}

\section{Material}

As matérias-primas utilizadas foram: leite integral, juntamente com o soro de leite líquido proveniente da fabricação de queijos, açúcar e bicarbonato de sódio.

\section{Produção dos doces}

O doce de leite foi produzido no laticínio do Instituto Federal de Educação, Ciência e Tecnologia do Sul de Minas Gerais - Campus Inconfidentes. Para sua produção, foram elaboradas seis formulações, enumeradas de 1 a 6 , sendo o leite parcialmente substituído por diferentes concentrações de soro, cujas porcentagens foram de $16,67 \%, 25 \%, 33,33 \%$, $41,67 \%, 50 \%$ e $58,33 \%$ respectivamente, do volume total produzido, equivalente a 60 litros por formulação.

\section{Análise sensorial}

As análises sensoriais foram realizadas no Instituto Federal de Educação, Ciência e Tecnologia do Sul de Minas Gerais - Campus Inconfidentes, nas quais utilizou-se cabines individuais, com 123 consumidores, cujas idades variaram de 14 a 25 anos, sendo $73,17 \%$ do sexo feminino (90 mulheres e 33 homens). As amostras foram inseridas em copos plásticos, com capacidade de 50 $\mathrm{mL}$, previamente codificados em algarismos de 3 dígitos a partir do delineamento em bloco completo balanceado, na quantidade aproximada de $15 \mathrm{~g}$ de doce de leite (McFIE, 1990). Distribuiu-se as amostras à temperatura ambiente. No início das análises, orientouse aos consumidores a ingestão de água no intervalo de cada amostra, para a retirada de resíduos alimentares das papilas gustativas, evitando, assim, possíveis interferências nas análises.

Avaliou-se os doces por meio do teste de aceitação, utilizando uma escala hedônica estruturada em nove pontos, com extremos de "desgostei muitíssimo" a "gostei muitíssimo", que correspondem respectivamente de um a nove, para os atributos: aparência, aroma, sabor, textura e impressão global (STONE; SIDEL, 2004). Também houve a avaliação quanto à intenção de compra das amostras, em uma escala hedônica estruturada de cinco pontos, com extremos de "certamente não compraria" a "certamente compraria" (MEILGAARD et al., 2004). Por fim, o teste de ideal foi realizado a fim de descrever as condições ideais de gosto doce e consistência do produto de acordo com os consumidores, em que 0 corresponde a idealidade do doce, e os extremos +4 e -4 apontam que o mesmo está 
extremamente mais ou menos longe do ideal, respectivamente (STONE; SIDEL, 2004).

\section{Análises físico-químicas}

As análises físico-químicas do doce de leite foram realizadas em triplicata no Instituto Federal de Educação, Ciência e Tecnologia do Sul de Minas Gerais - Campus Inconfidentes, nos Laboratórios de Solos, Bromatologia, Análise Sensorial, Processos Fermentativos e Tratamento de Resíduos do Núcleo de Alimentos e no Laticínio.

Para a determinação do teor de lipídeos das amostras de doce de leite utilizou-se o método de Gerber, que determina a quantidade de lipídio no leite e em seus derivados, sendo padronizado pelo método gravimétrico de Rose-Gottlieb. A análise de proteínas foi realizada através do método de Kjeldahl e o fator de conversão de nitrogênio total em proteína utilizado foi de 6,38 para produtos lácteos. A determinação de umidade foi realizada por meio de secagem das amostras em estufa à $105^{\circ} \mathrm{C}$, até peso constante. Mediuse o $\mathrm{pH}$ com o auxílio de um medidor de $\mathrm{pH}$ digital (Digimed modelo DM-22), diluindo-se as amostras em $100 \mathrm{~mL}$ de água e agitando-se a solução para melhor homogeneização. Essas análises seguiram metodologias do Instituto Adolfo Lutz (ZENEBON et al., 2008) para determinação.

Para a determinação dos sólidos solúveis totais $\left({ }^{\circ}\right.$ Brix) utilizou-se um refratômetro digital (Atago modelo PAL-1), com precisão de $\pm 0,2 \%$, e realizou-se a diluição das amostras na proporção de 1:1. O aparelho continha um tempo de leitura de 3 segundos para a obtenção do valor de Brix de cada amostra.

As análises colorimétricas foram realizadas com o auxílio de um colorímetro (Konica Minolta modelo CM-2300d) previamente calibrado, com placa de porcelana, utilizando o iluminante D65, empregando o sistema CIE $(\mathrm{L} * \mathrm{a} * \mathrm{~b} *)$, onde
$L^{*}$ representa a luminosidade, variando de preto a branco (0 a 100), a* varia de verde a vermelho (-120 a 120) e $b^{*}$ varia de azul a amarelo (-120 a 120).

\section{Análise de textura}

A análise do perfil de textura instrumental, ou Texture Profile Analysis (TPA), foi realizada utilizando-se o texturômetro Stable Micro Systems, modelo TA.XT2i. As amostras foram avaliadas no pote cilíndrico acrílico de $20 \mathrm{~mm}$ (P20) seguindo os parâmetros empregados por Rocha et al. (2012), que são a velocidade de pré-teste: 2 $\mathrm{mm} / \mathrm{s}$; velocidade de teste: $1 \mathrm{~mm} / \mathrm{s}$; velocidade de pós-teste: $2 \mathrm{~mm} / \mathrm{s}$; distância: $10 \mathrm{~mm}$; tempo: $5 \mathrm{~s}$; força de contato: $5 \mathrm{~g}$.

\section{Análise estatística}

As análises estatísticas, foram avaliadas utilizando a Análise de Variância - ANOVA e teste de média de Tukey $(\mathrm{p} \leq 0,05)$ por meio do software SensoMaker ${ }^{\circledR}$, assim como o mapa de preferência externo (PINHEIRO et al., 2013). As análises do teste de intenção de compra foram realizadas através de um histograma de distribuição de frequência, executado pelo Software Microsoft Excel ${ }^{\circledR}$.

\section{RESULTADOS E DISCUSSÃO}

Na Tabela 1, estão dispostos os valores médios dos cinco atributos sensoriais analisados: aparência, aroma, sabor, textura e impressão global, dos doces de leite com substituição parcial do leite por soro de leite, em diferentes concentrações.

Ao avaliar as médias hedônicas, encontrou-se os termos da escala de nove pontos, sendo estes: gostei ligeiramente (6) e gostei moderadamente (7) para os atributos aparência, aroma, sabor, textura e impressão global. 
Para o atributo aroma as amostras não diferiram entre si $(\mathrm{p}>0,05)$. Já para aparência, apenas as formulações com 16,67\%, 25\%, $33,33 \%$, e $41,67 \%$ de soro não diferiram entre si, ou seja, nas concentrações do soro de até no máximo 41,67\% observou-se que a aparência não foi afetada, porém as formulações com $50 \%$ e $58,33 \%$ diferiram entre as demais, demonstrando que concentrações mais elevadas de soro interferiram na aparência, para os provadores.

A formulação com $41,67 \%$ de soro apresentou o maior valor médio para o atributo sabor, comparada às outras formulações, sendo a mais aceita e também não diferindo das formulações $25 \%$ e $58,33 \%$. Para a textura a amostra de $41,67 \%$ apresentou um maior valor médio, assim como para o atributo sabor, porém a mesma não diferiu das formulações com $25 \%$ e $33,33 \%$.

Por fim, na impressão global, de modo geral, a formulação com 41,67\%, também obteve maior média do que as demais formulações, sendo que a mesma não diferiu das amostras com 16,67\%, 25\% e 33,33\%, porém em relação aos atributos analisados, as amostras $50 \%$ e $58,33 \%$ diferiram das demais, apresentando menor média em todas as suas características averiguadas, com exceção do atributo aroma.

A Tabela 2 apresenta os valores médios do teste do ideal para os atributos gosto doce e consistência.

Analisando a Tabela 2, nota-se que não houve diferença significativa $(\mathrm{p}>0,05)$ entre as amostras analisadas de doce de leite em relação ao gosto doce, portanto todas as amostras possuem gosto doce acima do ideal. Em contrapartida, quanto à consistência, a única amostra que diferiu das demais foi a $25 \%$, que apresentou o valor de média mais elevado.

$\mathrm{Na}$ Tabela 3, apresentou-se os valores médios e desvio padrão da composição centesimal (umidade, proteína, lipídeos e textura) dos doces de leite substituídos com soro. Pode-se observar que os valores de textura, apresentados nessa tabela, comparado com os dados acima (consistência), demonstram que a amostra 2 apresenta uma maior média em relação a esses dois parâmetros, porém quanto à Tabela 1, sensorialmente avaliada pelos provadores, esse atributo expressou uma média intermediária quanto ao mesmo requisito avaliado.

Tabela 1 - Valores médios* e desvio padrão dos atributos do teste de aceitação das amostras de doce de leite substituídos com soro de leite

\begin{tabular}{ccccccc}
\hline \multirow{2}{*}{ Amostras } & $\begin{array}{c}\text { \% de } \\
\text { soro }\end{array}$ & Aparência & Aroma & Sabor & Textura & $\begin{array}{c}\text { Impressão } \\
\text { Global }\end{array}$ \\
\hline 1 & 16,67 & $7,32 \pm 1,19^{\mathrm{a}}$ & $7,32 \pm 1,33^{\mathrm{a}}$ & $7,16 \pm 1,72^{\mathrm{b}}$ & $6,70 \pm 1,09^{\mathrm{b}}$ & $7,12 \pm 1,45^{\mathrm{ab}}$ \\
2 & 25,00 & $7,67 \pm 1,19^{\mathrm{a}}$ & $7,10 \pm 1,30^{\mathrm{a}}$ & $7,40 \pm 1,54^{\mathrm{ab}}$ & $7,05 \pm 1,71^{\mathrm{ab}}$ & $7,22 \pm 1,43^{\mathrm{ab}}$ \\
3 & 33,33 & $7,74 \pm 1,13^{\mathrm{a}}$ & $7,17 \pm 1,35^{\mathrm{a}}$ & $7,08 \pm 1,75^{\mathrm{b}}$ & $6,96 \pm 1,72^{\mathrm{ab}}$ & $7,15 \pm 1,51^{\mathrm{ab}}$ \\
4 & 41,67 & $7,93 \pm 1,25^{\mathrm{a}}$ & $7,46 \pm 1,23^{\mathrm{a}}$ & $7,74 \pm 1,23^{\mathrm{a}}$ & $7,57 \pm 1,32^{\mathrm{a}}$ & $7,57 \pm 1,20^{\mathrm{a}}$ \\
5 & 50,00 & $7,10 \pm 1,40^{\mathrm{b}}$ & $7,13 \pm 1,40^{\mathrm{a}}$ & $7,08 \pm 1,65^{\mathrm{b}}$ & $6,51 \pm 1,89^{\mathrm{b}}$ & $6,91 \pm 1,53^{\mathrm{b}}$ \\
6 & 58,33 & $6,75 \pm 1,69^{\mathrm{b}}$ & $7,00 \pm 1,39^{\mathrm{a}}$ & $7,22 \pm 1,45^{\mathrm{ab}}$ & $6,62 \pm 1,70^{\mathrm{b}}$ & $6,92 \pm 1,57^{\mathrm{b}}$ \\
\hline
\end{tabular}

* Valores médios (média \pm desvio-padrão) de três repetições. Letras diferentes na mesma coluna indicam diferença significativa (Tukey, $\mathrm{p} \leq 0,05$ ). 
Na Figura 1, estão ilustrados os dados de porcentagem de intenção de compra para cada formulação de doce de leite.

Analisando a Figura 1 de intenção de compra, verifica-se que as amostras 4 e 3 obtiveram as maiores frequências de intenções de compras positivas, que correspondem as respostas "certamente compraria" e "provavelmente compraria", totalizando, aproximadamente $68 \%$ e $60 \%$, respectivamente. A amostra 5 apresentou maior frequência $(33,5 \%)$ de indecisão na intenção de compra representada pelo termo "talvez compraria". As amostras 1, 5 e 6 exibiram maiores frequências de intenções de compras negativas que correspondem às atitudes de compra "provavelmente não compraria" e "certamente não compraria", correspondendo a $20 \%, 17 \%$ e $17 \%$, respectivamente. Desta forma, verifica-se que as formulações de doce de leite com concentrações intermediárias $(33,33 \%$ e $41,67 \%$ ) de soro obtiveram maior aceitação pelos provadores.

Tabela 2 - Valores médios* do teste de ideal para gosto doce e consistência para as amostras de doce de leite substituídos com soro

\begin{tabular}{cccc}
\hline Amostras & \% de soro & Gosto doce & Consistência \\
\hline 1 & 16,67 & $0,78^{\mathrm{a}}$ & $0,15^{\mathrm{b}}$ \\
2 & 25,00 & $0,61^{\mathrm{a}}$ & $0,77^{\mathrm{a}}$ \\
3 & 33,33 & $0,73^{\mathrm{a}}$ & $0,16^{\mathrm{b}}$ \\
4 & 41,67 & $0,45^{\mathrm{a}}$ & $-0,22^{\mathrm{b}}$ \\
5 & 50,00 & $0,89^{\mathrm{a}}$ & $0,05^{\mathrm{b}}$ \\
6 & 58,33 & $0,84^{\mathrm{a}}$ & $-0,24^{\mathrm{b}}$ \\
\hline
\end{tabular}

* Letras diferentes na mesma coluna indicam diferença significativa (Tukey, $\mathrm{p} \leq 0,05$ ).

Tabela 3 - Valores médios* e desvio padrão das características físico-químicas das amostras de doce de leite substituídos com soro de leite

\begin{tabular}{cccccc}
\hline Amostras & \% de soro & Umidade (\%) & Proteínas (\%) & Lipídeos (\%) & Textura (N) \\
\hline 1 & 16,67 & $44,67 \pm 1,15^{\mathrm{a}}$ & $7,33 \pm 0,06^{\mathrm{a}}$ & $10,33 \pm 1,15^{\mathrm{ab}}$ & $0,19 \pm 0,0^{\mathrm{c}}$ \\
2 & 25,00 & $25,00 \pm 0,58^{\mathrm{b}}$ & $6,90 \pm 0,17^{\mathrm{b}}$ & $10,67 \pm 0,58^{\mathrm{ab}}$ & $0,34 \pm 0,0^{\mathrm{a}}$ \\
3 & 33,33 & $44,00 \pm 0,58^{\mathrm{a}}$ & $6,73 \pm 0,06^{\mathrm{b}}$ & $12,33 \pm 0,58^{\mathrm{a}}$ & $0,22 \pm 0,0^{\mathrm{b}}$ \\
4 & 41,67 & $44,33 \pm 1,53^{\mathrm{a}}$ & $6,20 \pm 0,10^{\mathrm{c}}$ & $10,67 \pm 1,53^{\mathrm{ab}}$ & $0,18 \pm 0,00^{\mathrm{c}}$ \\
5 & 50,00 & $28,33 \pm 0,00^{\mathrm{b}}$ & $5,80 \pm 0,00^{\mathrm{d}}$ & $9,00 \pm 0,00^{\mathrm{bc}}$ & $0,11 \pm 0,03^{\mathrm{d}}$ \\
6 & 58,33 & $35,00 \pm 0,00^{\mathrm{ab}}$ & $5,70 \pm 0,26^{\mathrm{d}}$ & $7,00 \pm 0,00^{\mathrm{c}}$ & $0,10 \pm 0,00^{\mathrm{d}}$ \\
\hline
\end{tabular}

* Valores médios (média \pm desvio-padrão) de três repetições. Letras diferentes na mesma coluna indicam diferença significativa (Tukey, $\mathrm{p} \leq 0,05$ ). 
Identifica-se que o doce de leite com maior intenção de compra (amostra 4) foi o mesmo que obteve a maior média para sabor e textura, portanto, entende-se que essas características organolépticas são um diferencial na decisão de compra de um produto.

Os padrões físico-químicos para o doce de leite, conforme a Portaria $n^{\circ} 354$ de 04 de setembro de 1997 (BRASIL, 1997) são de no máximo $30 \%$ para umidade. O conteúdo de matéria gorda deve estar entre 6,0 e 9,0\% de produto e o de proteína deve ser de no mínimo 5,0\%.

Em relação aos dados de umidade, podese observar que houve diferença significativa das amostras $2(25 \%)$ e $5(50 \%)$ em relação às demais, sendo que estas apresentaram os menores valores para este parâmetro, verificando-se que somente as mesmas estão dentro do limite estabelecido pela legislação. Segundo Demiati et al. (2001), a baixa umidade do doce de leite pastoso melhora a conservação do produto, porém facilita o aparecimento de arenosidade, um defeito percebido sensorialmente que é comum em doces de leite.

No estudo realizado por Machado (2005), citado por Madrona et al., (2009) também foram encontrados valores de umidades relativamente altas, entre $34,00 \%$ e $39,86 \%$, o que pode ser explicado pelo soro de leite apresentar maior quantidade de água se comparado ao leite. Porém, neste estudo, as formulações com $25 \%$ e $50 \%$ não diferiram entre si, apresentando os menores valores de umidade, mesmo com o dobro da concentração de soro presente na amostra com $50 \%$, em relação à amostra com $25 \%$, enquanto a amostra contendo $58,33 \%$ de soro, apresentou valor médio de umidade intermediário. A dispersão de dados em relação a umidade pode ser decorrente do processo manual de fabricação do doce de leite, o que impede a padronização adequada do processo, podendo então ter variação no tempo de cocção, que segundo Demiate et al. (2001), juntamente ao tipo de equipamento utilizado interfere diretamente na umidade do produto final, devido a retirada de água

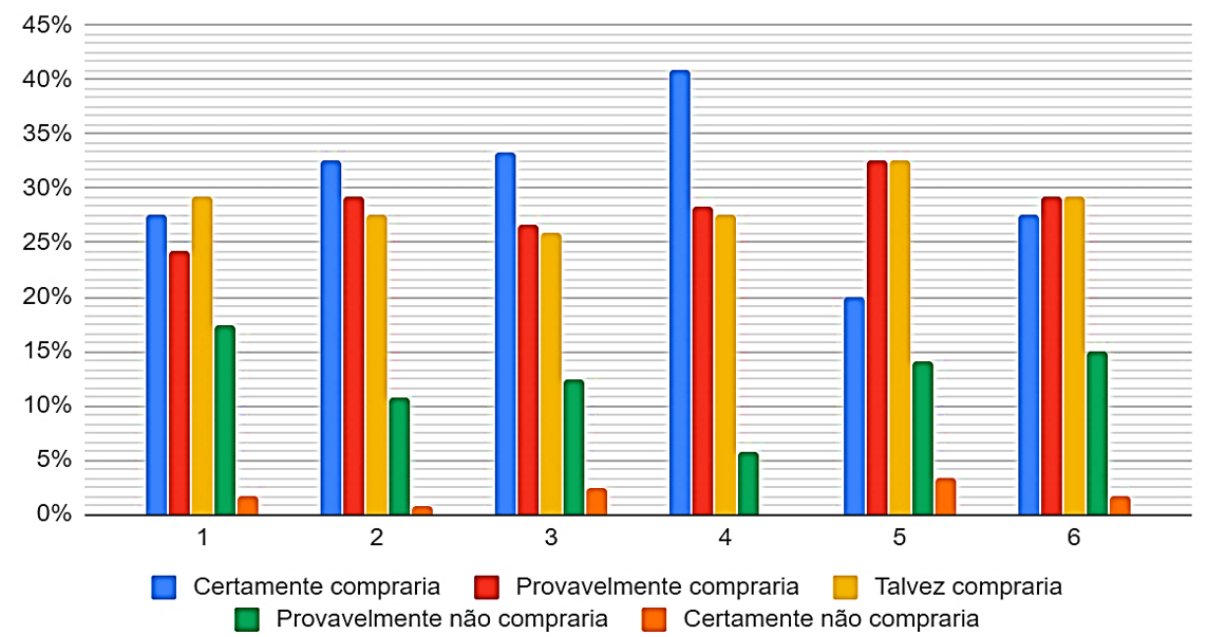

Figura 1 - Percentual de intenção de compra para as formulações de doce de leite $(1-16,67 \%$; $2-25 \% ; 3-33,33 \% ; 4-41,67 \% ; 5-50 \% ; 6-58,33 \%$ de soro) 
do leite através da transferência de energia na forma de calor com vapor indireto.

O aumento de soro de leite na formulação resultou em uma queda nos valores de proteínas. O soro utilizado foi oriundo do processo de fabricação de queijos, o que justificaria o menor teor de proteínas nos doces com maior quantidade de soro, pois segundo Haraguchi et al. (2005), proteínas do leite são extraídas durante o processo de fabricação do queijo, ou seja, sua maior parte fica retida no queijo, resultando em uma menor concentração no soro de leite.

Para gordura, pode-se observar que a amostra de doce de leitecom 33,33\% de soro não apresentou diferença significativa para este atributo, em relação às amostras 16,67\%, $25 \%$ e $41,67 \%$, porém as amostras com $50 \%$ e $58,33 \%$ diferiram das demais, apresentando um valor médio menor. Com isso, observa-se que quanto maior a concentração de soro, menor será a quantidade de gordura, visto que esta está presente em maior quantidade no leite do que no soro. Além disso, estes teores apresentados foram os únicos que estiveram dentro do limite estabelecido pela legislação.

Para a textura, a amostra contendo $25 \%$ de soro apresentou uma maior média quando relacionada com as demais, fato que pode ter ocorrido devido a sua quantidade de gordura, pois ambos parâmetros não diferiram significativa entre si $(\mathrm{p}>0,05)$. Segundo Demiate et al. (2001), o teor de lipídios auxilia na viscosidade do produto, automaticamente afetando a textura, pois já que o leite é de origem animal, ele contém gordura saturada, e esta se encontra em forma sólida (COSTA; PELUZIO, 2008), podendo causar assim, uma maior consistência ao doce. Já as amostras 50\% e 58,33\% tiveram um baixo valor na textura, pois elas apresentaram maior concentração de soro de leite e consequentemente um menor teor de gordura.

A Tabela 4 apresenta as médias e os desvios padrões encontrados para os sólidos solúveis e os valores de $\mathrm{pH}$ das amostras.

$\mathrm{Na}$ Tabela 4 é possível notar que a amostra 5 apresentou maior média no que se refere a sólidos solúveis, porém, a mesma não apresentou diferença significativa da amostra 6. A amostra 1 foi a que conteve a menor média, isso acontece porque o soro de leite é rico em lactose resultando em uma maior concentração de ${ }^{\circ}$ Brix no doce.

Para os dados de $\mathrm{pH}$, observa-se que as amostras de doce de leite com $41,67 \%$ a $50 \%$ de soro não diferiram da amostra com 58,33\%, enquanto que a mesma diferiu-se das demais. Contudo, todos os valores encontrados de $\mathrm{pH}$ ficaram entre 6 e 7, ou seja, próximo ao meio ácido e neutro, o que segundo Brião et al. (2011) citado por Francisquini (2016), é um fator determinante para a reação de Maillard.

A Tabela 5 apresenta as médias e o desvio padrão obtidos na análise de cor das amostras de doce de leite substituído por soro, sendo que o termo $\mathrm{L}^{*}$ representa a luminosidade, $\mathrm{a}^{*}$ a coordenada verde/ vermelho ( $+\mathrm{a}$ indica vermelho $\mathrm{e}-\mathrm{a}$ indica verde) e b* a coordenada amarelo/azul $(+b$ indica amarelo e $-\mathrm{b}$ indica azul).

Para a coordenada $\mathrm{a}^{*}$, as amostras $1 \mathrm{e}$ 4 não apresentaram diferença significativa, sendo as maiores médias, e as amostras 2, 3, 5 e 6 também não diferiram entre si, apresentando menores médias. Todas as médias foram positivas, o que indica que os comprimentos de onda da luz detectados estão associados à cor vermelha. Para a coordenada b*, a amostra 4 apresentou maior média e as amostras 2 e 5 as menores, não apresentando diferença significativa. Nota-se que todas médias foram positivas indicando uma cor amarelada que é característica deste produto. Em relação a luminosidade, nota-se que a amostra 1 apresentou maior média em relação às demais, o que evidencia ser o doce mais claro, já as amostras 2,5 e 6 não diferiram entre si, apresentando as menores médias.

A cor escura do doce de leite é resultante 
da reação de Maillard que, segundo Ferreira et al. (2012), pode ser influenciada pela acidez do leite, a quantidade e o momento da adição do bicarbonato de sódio, assim como a presença de açúcares redutores além da lactose, o teor inicial e final de sólidos solúveis, o tempo gasto para a evaporação, que varia entre uma hora e quinze minutos e uma hora e trinta minutos, e a pressão de vapor utilizada nos tachos. Dentre esses fatores, os que podem ter interferido na coloração dos doces de leite são: tempo de evaporação, uma vez que não houve o controle do tempo de cozimento, e o pH, já que a amostra 1, que apresentou coloração mais clara, também foi a que apresentou o menor valor de $\mathrm{pH}$.

O mapa de preferência externo (Figura 2) representa a relação da variável "impressão global" com os valores das análises físicoquímicas discutidas anteriormente através da dispersão de pontos.

A partir desse gráfico é possível notar

Tabela 4 - Valores médios* e desvio padrão encontrados para os teores de sólidos solúveis ( ${ }^{\circ}$ Brix $)$ e os valores de $\mathrm{pH}$ das amostras

\begin{tabular}{cccc}
\hline Amostras & \% de soro & ${ }^{\circ}$ Brix & $\mathrm{pH}$ \\
\hline 1 & 16,67 & $57,93 \pm 1,22^{\mathrm{d}}$ & $6,57 \pm 0,0^{\mathrm{d}}$ \\
2 & 25,00 & $65,27 \pm 0,58^{\mathrm{bc}}$ & $6,74 \pm 0,02^{\mathrm{b}}$ \\
3 & 33,33 & $64,87 \pm 2,38 \mathrm{bc}$ & $6,66 \pm 0,02^{\mathrm{c}}$ \\
4 & 41,67 & $62,67 \pm 0,3^{\mathrm{c}}$ & $6,82 \pm 0,03^{\mathrm{a}}$ \\
5 & 50,00 & $68,73 \pm 0,81^{\mathrm{a}}$ & $6,81 \pm 0,02^{\mathrm{a}}$ \\
6 & 58,33 & $67,60 \pm 0,80^{\mathrm{ab}}$ & $6,79 \pm 0,03^{\mathrm{ab}}$ \\
\hline
\end{tabular}

* Valores médios (média \pm desvio-padrão) de três repetições. Letras diferentes na mesma coluna indicam diferença significativa (Tukey, $\mathrm{p} \leq 0,05$ ).

Tabela 5 - Valores médios* e desvio padrão dos parâmetros instrumentais de cor dos doces de leite substituídos com soro

\begin{tabular}{cccc}
\hline Amostras & $\mathrm{L}^{*}$ & $\mathrm{a}^{*}$ & $\mathrm{~b}^{*}$ \\
\hline 1 & $75,79 \pm 0,60^{\mathrm{a}}$ & $5,27 \pm 0,13^{\mathrm{a}}$ & $29,99 \pm 0,23^{\mathrm{ab}}$ \\
2 & $64,39 \pm 2,14^{\mathrm{b}}$ & $3,58 \pm 0,07^{\mathrm{b}}$ & $26,55 \pm 0,8^{\mathrm{c}}$ \\
3 & $70,61 \pm 6,31^{\mathrm{ab}}$ & $4,04 \pm 0,61^{\mathrm{b}}$ & $28,22 \pm 0,3^{\mathrm{abc}}$ \\
4 & $70,19 \pm 2,18^{\mathrm{ab}}$ & $5,1 \pm 0,14^{\mathrm{a}}$ & $30,23 \pm 1,29^{\mathrm{a}}$ \\
5 & $66,53 \pm 2,05^{\mathrm{b}}$ & $3,6 \pm 0,04^{\mathrm{b}}$ & $27,05 \pm 0,84^{\mathrm{c}}$ \\
6 & $66,26 \pm 1,29^{\mathrm{b}}$ & $4,02 \pm 0,15^{\mathrm{b}}$ & $28,00 \pm 0,55^{\mathrm{bc}}$ \\
\hline
\end{tabular}

* Valores médios (média \pm desvio-padrão) de três repetições. Letras diferentes na mesma coluna indicam diferença significativa (Tukey, $\mathrm{p} \leq 0,05$ ). 
uma maior quantidade de vetores direcionados as amostras 3, $4 \mathrm{e} \mathrm{1}$, respectivamente, seguidas pela 5 e 6 , o que indica que essas amostras demonstraram maior aceitabilidade sensorial pelos consumidores.

A aceitabilidade pode estar relacionada ao maior teor de lipídeos presente na amostra 3 , considerando que este é um potencial fator determinante para a preferência, devido a capacidade dos lipídios em carrear compostos voláteis relevantes ao sabor e conferirem uma melhor textura aos produtos.

As amostras 1 e 4 , tendem a sua preferência aos valores correlacionados a luminosidade, fator este essencial para provocar o desejo de compra nos consumidores, em se tratando de um produto com altos valores de ${ }^{\circ}$ Brix, e seu aspecto pastoso, atributo bastante desejado. Já as amostras 5 e 6 podem estar associadas predominantemente a preferência por maiores valores de ${ }^{\circ}$ Brix e a aceitabilidade do público avaliador em relação a valores de $\mathrm{pH}$ mais próximos da neutralidade, em se tratando deste tipo de alimento.

\section{CONCLUSÕES}

A amostra com melhor aceitação sensorial foi a amostra 4, diferindo significativamente apenas quanto ao atributo sabor em relação a amostra 3 , que por sua vez foi a mais aceita segundo o mapa de preferência. $\mathrm{O}$ intervalo de maior aceitação esteve entre os valores intermediários de substituição de leite por soro de leite, destoando apenas para os intervalos com maiores quantidades de soro, que mesmo apresentando diferença significativa em muitos atributos demonstraram maior aceitação que a amostra 1 ,com menor quantidade de soro, pelo mapa de preferência.

Diante destes resultados, é possível afirmar que a substituição parcial de leite por soro de leite é uma alternativa viável dentro do intervalo compreendido pelas amostras mais aceitas, nas condições realizadas neste estudo.

\section{REFERÊNCIAS}

ALVES, M. P. et al. Soro de leite: Tecnologias

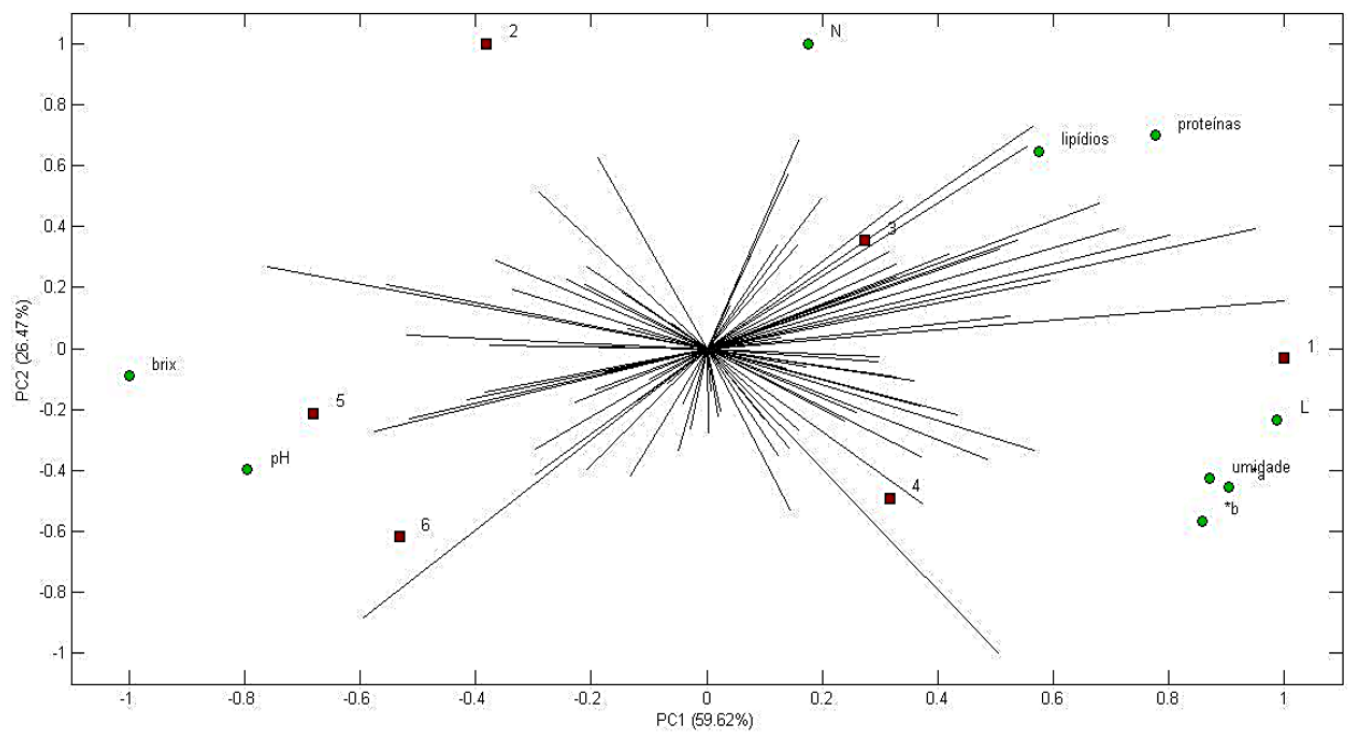

Figura 2 - Mapa de preferência externo para as amostras de doce de leite 
para o processamento de coprodutos. Revista Instituto de Laticínios Cândido Tostes, v. 69, n. 3, p. 212-226, 2014.

BRASIL. Ministério da Agricultura e do Abastecimento. Portaria $n^{\circ} 354$, de 4 de setembro de 1997. Regulamento Técnico para Fixação de Identidade e Qualidade de Doce de Leite. Diário Oficial da República Federativa do Brasil: seção 1, Brasília, DF, n. 172 , p. $19.685,08$ set. 1997.

BRIÃO, V. B. et al. Cinética do escurecimeno não-enzimático com soluções modelo de açúcares e aminoácidos em pH neutro e ácido. Acta Scientiarum Technology, v. 33, n. 1, p. 87-93, 2011.

Costa, N. M. B.; PEluziO, M. C. G. Nutrição Básica e Metabolismo. 1. ed. Viçosa: Editora UFV, 2008. 400 p.

DEMIATE, I. M.; KONKEL, F. E.; PEDROSO, R. A. Avaliação da qualidade de amostras comerciais de doce de leite pastoso - composição química. Ciência e Tecnologia de Alimentos, v. 21, n. 1, p. 108-114, 2001.

FEIHRMANN, A. C.; CICHOSKI, A. J.; REZENDE, D. F. Doce de leite (revisão). Higiene Alimentar, v. 18, n. 118, p. 21-23, 2004

FERRARI, A. S.; BALDONI, N. R.; AZEREDO, E. M. C. Análise sensorial e físico-química de produtos elaborados à base de soro de leite. Revista da Universidade Vale do Rio Verde, v. 11, n. 1, p.216-223, 2013.

FERREIRA, L. O. et al. Avaliação das características de qualidade de doces de leite comerciais. Revista Instituto de Laticínios Cândido Tostes, v. 67, n. 387, p. 5-11, 2012.
FRANCISQUINI, J. D'A. Caracterização e avaliação de indicadores físico-químicos, tecnológicos e de tratamento térmico em doces de leite caracterização e avaliação de indicadores físico-químicos, tecnológicos e de tratamento térmico em doces de leite. 2016. Dissertação (Mestrado em Ciência e Tecnologia do Leite e Derivados) - Faculdade de Farmácia, Universidade Federal de Juiz de Fora, Juiz de Fora, 2016.

HARAGUCHI, F. K.; ABREU, W. C.; PAULA, H. Proteínas do soro do leite: Composição, propriedades nutricionais, aplicações no esporte e benefícios para a saúde humana. Revista de Nutrição, v. 1, n. 2, p. 1-16, 2005.

MACHADO, L. M. P. Uso de soro de queijo e amido de milho modificado na qualidade do doce de leite pastoso. 2005. 170 f. Tese (Doutorado em Tecnologia de Alimentos) - Faculdade de Engenharia de Alimentos, Universidade Estadual de Campinas, Campinas, 2005.

MADRONA, G. S. et al. Estudo do efeito da adição de soro de queijo na qualidade sensorial do doce de leite pastoso. Ciência e Tecnologia de Alimentos, v. 4, n. 29, p. 826-833, 2009.

McFIE, H. J. H. Assessment of the sensory properties of food. Nutrition Reviews, v. 48, n. 2, p.87-93, 1990.

MEILGAARD, M. C.; CIVILLE, G.; CARR, T. Sensory Evaluation Techniques. 3. ed. New York: Boca Raton, 2004. 387 p.

PINHEIRO, A. C. M.; NUNES, C. A.; VIETORIS, V. SensoMaker: A tool for sensorial characterization of food products. Ciência e Agrotecnologia, v. 37, n. 3, p. 199-201, 2013. 
ROCHA, L. O. F. et al. Avaliação físicoquímica e sensorial de doce de leite elaborado com extrato hidrossolúvel de soja e soro de leite sabor café. Revista Brasileira de Produtos Agroindustriais, v. 14, n. 3, p. 251-259, 2012.

SGARBIER, V. C. Propriedades fisiológicasfuncionais das proteínas do soro de leite. Revista de Nutrição, v. 4, n. 17, p. 397-409, 2004.

SILVA, C. E. S.; CARVALHO, N. C.; GONÇALVES, T. C. C. O desenvolvimento do produto: Soro de leite bovino no combate à desnutrição. In: ENCONTRO NACIONAL DE ENGENHARIA DE PRODUÇÃO, 23.,
2003, Ouro Preto. Anais [...] Ouro Preto: ABEPRO, 2003.

STONE, H.; SIDEL, J. Sensory Evaluation Practices. 3. ed. New York: Academic Press, 2004. 408 p.

TEIXEIRA, L. V. Análise sensorial na indústria de alimentos. Revista do Instituto de Laticínios Cândido Tostes, v. 64, n. 366, p. 12-21, 2009.

ZENEBON, O.; PASCUET, N. S.; TIGLEA, P. (coord.). Métodos físico-químicos para análise de alimentos. 4. ed., 1. ed. digital. São Paulo: Instituto Adolfo Lutz, 2008.1020 p. 